удК

\title{
УЛУЧШЕНИЕ ХАРАКТЕРИСТИК ПРЯМОУГОЛЬНОЙ МИКРОПОЛОСКОВОЙ АНТЕННЫ С ПОМОЩЬЮ ДВОЙНОГО Н-ОБРАЗНОГО МЕТАМАТЕРИАЛА
}

\author{
ПРИТ КАУР ${ }^{1}$, С. К. АГАРВАЛ ${ }^{1}$, АСОК ДЕ $^{2}$ \\ ${ }^{1}$ ҮМСА университет науки и технологий, \\ Индия, Фаридабад, Харьяна \\ ${ }^{2}$ Национальный технологический институт, \\ Индия, Патна
}

\begin{abstract}
Аннотация. В статье описана разработанная прямоугольная микрополосковая антенна (ПМА) с использованием двойного Н-образного метаматериала. Вначале разработан и оптимизирован двойной Н-образный метаматериал для резонансной частоты микрополосковой антенны 5,2 ГГц. Установлено, что включение этого метаматериала в подложку под антенной базовой конфигурации улучшает ее обратные потери и рабочую полосу частот без изменения резонансной частоты и коэффициента усиления. Для дальнейшего повышения коэффициента усиления и коэффициента полезного действия ПМА со встроенным метаматериалом в структуру антенны добавлена надложка из двойного Н-образного метаматериала на расстоянии $\lambda / 3$ над антенной. В результате получена ПМА с использованием метаматериалов, которая обеспечивает высокий коэффициент усиления, широкую рабочую полосу частот и хорошее согласование сопротивления. Предложенная антенна смоделирована и оптимизирована с помощью программного обеспечения HFSS. Изготовлен опытный образец антенны и результаты измерений характеристик этой антенны хорошо согласуются с результатами моделирования.
\end{abstract}

Ключевые слова: метаматериал; прямоугольная микрополосковая антенна; ПМА; коэффициент отражения

\section{1. ВВЕДЕНИЕ}

В современных беспроводных и электронных системах значительно повышается потребность в компактных, широкополосных, недорогих и легких излучателях с высоким коэффициентом усиления и простой системой подачи питания $[1,2]$. Обычная прямоугольная микрополосковая антенна (ПМА), которая используется в беспроводных приложениях, имеет низкий профиль, простоту конструкции, низкую стоимость, простоту изготовления и простую систему подачи питания, однако эта антенна имеет низкий коэффициент усиления и узкую рабочую полосу частот из-за влияния поверхностной волны на ее диаграмму излуче- ния [3]. Для решения этой проблемы возможно использовать решетку из микрополосковых антенн, но ее применение ограничивается сложной системой подачи питания и более низким коэффициентом полезного действия.

Недавно исследователями предложено использование метаматериала для разработки антенн с улучшенными характеристиками и пониженным профилем [1]. Метаматериалы являются искусственными материалами, которые образованы путем внедрения специфических включений в материал-основу и могут быть спроектированы таким образом, чтобы обеспечивать требуемые электромагнитные свойства. 


\section{БИБЛИОГРАФИЧЕСКИЙ СПИСОК}

1. Metamaterial superstrate and electromagnetic band-gap substrate for high directive antenna / Huiliang Xu, Zeyu Zhao, Yueguang Lv, Chunlei Du, Xiangang Luo // Int. J. Infrared Millimeter Waves. - 2008. - Vol. 29, No. 5. - P. 493-498. - DOI : 10.1007/s10762-0089344-y.

2. Planar and cylindrical metamaterial structures for antenna applications / A. Chauraya, J. Kelly, G. K. Palikaras, C. B. Mulenga, J. A. Flint, A. P. Feresidis, J. C. Vardaxoglou // EMTS Int. URSI Commision B-Electromagnetic Theory Symp. July 2007.

3. Liang $J$. Radiation characteristics of a microstrip patch over an electromagnetic bandgap surface / Jing Liang, Hung-Yu David Yang // IEEE Trans. Antenna 
Propag. — Jun. 2007. — Vol. 55, No. 6. - P. 1691-1697. — DOI : $\underline{10.1109 / \text { TAP.2007.898633. }}$.

4. Kock W. P. Metal-lens antennas / W. P. Kock // Proc. IRE. - Nov. 1946. - Vol. 34, No. 11. - P. 828-836. - DOI : 10.1109/JRPROC.1946.232264.

5. A study of using metamaterials as antenna substrate to enhance gain / B.-I. Wu, W. Wang, J. Pacheco, X. Chen, T. M. Grzegorczyk, J. A. Kong // PIER. - 2005. Vol. 51. - P. 295-328. — DOI : 10.2528/PIER04070701.

6. Near-field plane-wave-like beam emitting antenna fabricated by anisotropic metamaterial / Y. G. Ma, P. Wang, X. Chen, C. K. Ong // Appl. Phys. Lett. — 2009. Vol. 94. — P. 044107. — DOI : $10.1063 / 1.3077128$.

7. Rectangular microstrip patch antenna with "pentagonal rings" shaped metamaterial cover / Bimal Garg, Nitin Agrawal, Vijay Sharma, Ankita Tomar, Prashant Dubey // Communication Systems and Network Technologies : Int. Conf. CSNT, 11-13 May 2012, Rajkot : proc. - IEEE, 2012. - P. 40-44. - DOI : 10.1109/ CSNT.2012.18.

8. A compact multi-split ring resonator loaded antenna / R. Pandeeswari, S. Raghavan, Pravin A. Bagde, Ananda Kumar Chittipothul // Communications and Signal Processing : Int. Conf. ICCSP, 3-5 Apr. 2013, Melmaruvathur : proc. - IEEE, 2013. - P. 807-810. DOI : $10.1109 /$ iccsp.2013.6577168.

9. Hybrid mode wideband patch antenna loaded with a planar metamaterial unit cell / Jaegeun Ha, Kyeol Kwon, Youngki Lee, Jaehoon Choi // IEEE Trans. Antennas Propag. - Feb. 2012. - Vol. 60, No. 2. - P. 1143-1147. — DOI : 10.1109/TAP.2011.2173114.

10. A low-cost compact patch antenna with beam steering based on CSRR-loaded ground / Wenquan Cao, Yang Xiang, Bangning Zhang, Aijun Liu, Tongbin Yu, Daosheng Guo // IEEE Antennas Wireless Propag. Lett. - 2011. - Vol. 10. - P. 1520-1523. - DOI : $\underline{10.1109 /}$ LAWP.2011.2181316.

11. Attia $H$. Artificial magneto-superstrates for gain and efficiency improvement of microstrip antenna arrays / H. Attia, O. Siddiqui, O. M. Ramahi // Progress in Electromagnetics Research Symp., 5-8 Jul. 2010, Cambridge, USA : proc. — 2010. — P. 878-881.
12. Palandoken M. Broadband microstrip antenna with left-handed metamaterials / Merih Palandoken, Andre Grede, Heino Henke // IEEE Trans. Antennas Propag. - Feb. 2009. - Vol. 57, No. 2. - P. 331-338. - DOI : 10.1109/TAP.2008.2011230.

13. Kaur $P$. Design of compact rectangular patch antenna using square grid and I shaped metamaterial / Preet Kaur, S. K. Aggarwal, Asok De // Signal Processing and Communication : Int. Conf. ICSC, 16-18 Mar. 2015, Noida : proc. — IEEE, 2015. — P. 132-135. — DOI : 10.1109/ICSPCom.2015.7150634.

14. Blaha M. Planar resonators for metamaterials / Michal Blaha, Jan Machac // Radioengineering. - Sept. 2012. - Vol. 21, No. 3. - P. 852-859. - URL : http://www.radioeng.cz/fulltexts/2012/12 $03 \quad 0852 \quad 085$ 9.pdf.

15. Kaur P. Double H shaped metamaterial embedded compact RMPA / Preet Kaur, S. K. Aggarwal, Asok De // Advances in Computing, Communications and Informatics : Int. Conf. ICACCI, 24-27 Sept. 2014, New Delhi : proc. — IEEE, 2014. — P. 483-486. — DOI : 10.1109/ICACCI.2014.6968492.

16. Pozar D. M. Microstrip Antennas: The Analysis and Design of Microstrip Antennas and Arrays / David M. Pozar, Daniel H. Schaubert. - NewYork : IEEE Press, 1995. - DOI : $10.1109 / 9780470545270$.

17. Nicolson A. M. Measurement of the intrinsic properties of materials by time-domain techniques / A. M. Nicolson, G. F. Ross // IEEE Trans. Instrum., Meas. Nov. 1970. - Vol. 19, No. 4. - P. 377-382. - DOI : 10.1109/tim.1970.4313932.

18. Weir W. B. Automatic measurement of complex dielectric constant and permeability at microwave frequencies / W. B. Weir // Proc. IEEE. - Jan. 1974. Vol. 62, No. 1. - P. 33-36. — DOI : 10.1109/PROC. 1974.9382 .

19. Ziolkowski R. W. Design fabrication, and testing of double negative metamaterials / R. W. Ziolkowski // IEEE Trans. Antennas Propag. - Jul. 2003. - Vol. 51, No. 7. - P. 1516-1529. - DOI : 10.1109/TAP.2003. $\underline{813622 .}$. 